\title{
Structural Effects on Mesomorphic Properties in Isomeric Liquid Crystalline Polyesters
}

\author{
Annino Sante Angeloni, Daniele Caretti, Michele Laus, ${ }^{*}$ \\ Emo CHIELLINI, ${ }^{* *, \dagger}$ and Giancarlo GALLI** \\ Dipartimento di Chimica Industriale e dei Materiali, Universita' di Bologna, \\ Viale Risorgimento 4, 40136 Bologna, Italy \\ *Dipartimento di Ingegneria Meccanica, Universita' di Brescia, \\ Via Valotti 9, 25060 Brescia, Italy \\ **Dipartimento di Chimica e Chimica Industriale, Universita' di Pisa, \\ Via Risorgimento 35, 56100 Pisa, Italy
}

(Received August 3, 1988)

\begin{abstract}
Two series (I-m and II-m) of isomeric liquid crystalline polyesters containing two mesogenic $p$-oxybenzoyl dyads separated by a couple of spacers derived from triethylene glycol and a polymethylene segment of variable length have been prepared and characterized for their mesomorphic properties. In both polymer series the parity and length of the polymethylene spacer play an integral role in determining the onset and stability of the liquid crystalline state. The thermodynamic data indicate that even members possess a great propensity to participate in the ordering process in the nematic state. Low mesogen order and depressed transition temperatures are observed for odd members of series II-m while no liquid crystalline behavior is detected for odd members of series I-m.

KEY WORDS $p$-Oxybenzoyl Dyads / Alternating Copolymers / Thermotropic Liquid Crystalline Polymers / Polymethylene Spacers / Polyoxyethylene Spacers /
\end{abstract}

The thermotropic mesomorphism of polymers comprising rod-like units joined by flexible segments is established in a variety of systems. ${ }^{1}$ However, the control by structural entities of the onset and stability of mesophases over the isotropic state is not yet fully understood. The critical interplay among the nature and shape of the rigid group, the junction of the spacer with the core and the conformational characteristics of the flexible segment result in a great sensitivity of the liquid crystalline properties of the polymeric system to minor changes in molecular structure. A fruitful approach for ascertaining the property-determining structural features consists of modifying the liquid crystalline proper- ties in homologous series of polymers. Following our interest $\mathrm{t}^{2-4}$ in developing liquid crystalline polymers with predetermined properties, in the present work we describe the mesomorphic characteristics of two series of polymers constituted by isomeric repeating units.

Both polymer repeating units contain two mesogenic $p$-oxybenzoyl dyads separated by a spacer derived from triethylene glycol and a polymethylene segment of variable length which are alternatively connected to the rigid core via an ether or ester moiety. As a result the functional groups connecting the flexible spacers with the p-oxybenzoyl dyad are varied in a complementary manner. The two

\footnotetext{
† To whom all correspondence should be addressed.
} 

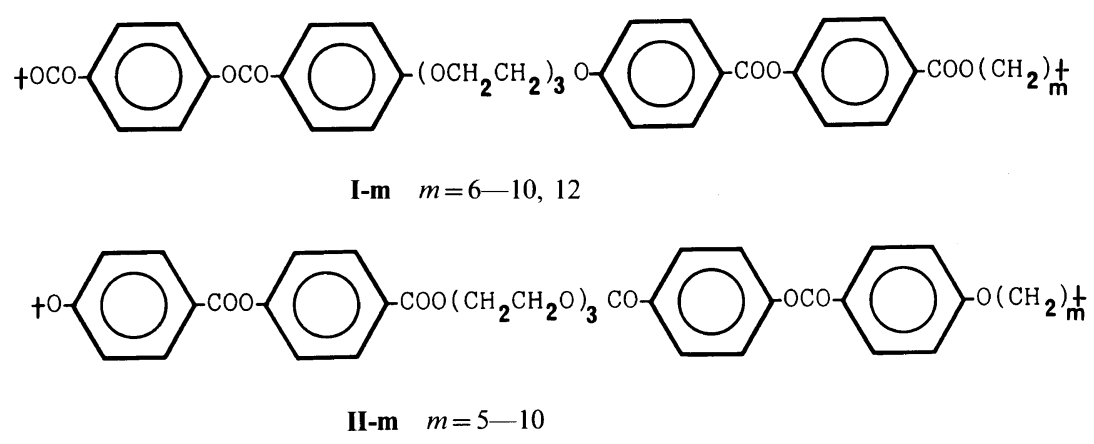

Scheme 1. Representation of structural formulas of the two studied series of isomeric semiflexible polyesters with twin structural units.

series of polyesters are designated as $\mathbf{I}-\mathbf{m}$ and II-m according to the different relative orientational placement of the mesogens and to the number $m$ of methylene units in the polymethylene spacer (Scheme 1).

\section{EXPERIMENTAL}

The synthesis of the polyesters and corresponding precursors will be described in detail in a forthcoming paper. ${ }^{5}$

Intrinsic viscosity measurements were performed in chloroform at $30^{\circ} \mathrm{C}$. Average molecular weights of polymers were obtained by gel permeation chromatography by a liquid chromatograph consisting of a Waters 590 pump, a Waters U6K injector and a Waters R-401 differential refractometer, equipped with a Shodex KF-804 column. Monodisperse polystyrene standards (Waters) were used for calibration. Differential scanning calorimetry (DSC) analyses were carried out under dry nitrogen flow on polymer samples (8-12 mg) with a Perkin Elmer DSC-7 calorimeter equipped with a 3700 data station using a Delta series standard program. Indium was employed for temperature and enthalpy calibration. The maximum in DSC enthalpic peaks with heating rate of $10 \mathrm{~K}$ $\min ^{-1}$ was taken as the phase transition temperature. Textures observations were performed by a Reichert Polyvar polarizing micros- cope equipped with a programmable Mettler FP52 hot stage at a scanning rate of $10 \mathrm{~K} \mathrm{~min}^{-1}$. X-Ray diffraction measurements were performed in a transmission mode by means of a conventional X-ray powder diffractometer. The Ni-filtered $\mathrm{Cu} K_{\alpha}$ radiation $(\lambda=1.54 \AA)$ was used.

\section{RESULTS AND DISCUSSION}

Polymer samples I-m and II-m were prepared by reacting 4,4'-dichloroformyl- $\alpha, \omega$ diphenylenetriethyleneoxide and 4,4'-dichloroformyl- $\alpha, \omega$-diphenoxyalkanes with $\operatorname{di}(4-$ hydroxybenzoate) alkanes and di(4-hydroxybenzoate) triethyleneoxide respectively (Scheme 2).

The polyesterification reaction was carried out by phase transfer polycondensation between the sodium salt of the diphenol in water and the corresponding diacid chloride in 1,2dichloroethane in the presence of benzyl tributylammonium bromide. In all cases the yields were nearly quantitative. The polymers have intrinsic viscosity values in the range $|\eta|=0.27-0.83 \mathrm{dlg}^{-1}$ to which average degrees of polymerization DPn $\geqslant 10$ correspond (Table I). With reference to recent results ${ }^{6}$ with structurally similar poly(ester- $\beta$-sulphide)s we assume that liquid crystalline properties are not affected in a substantial manner by the molecular weight. 

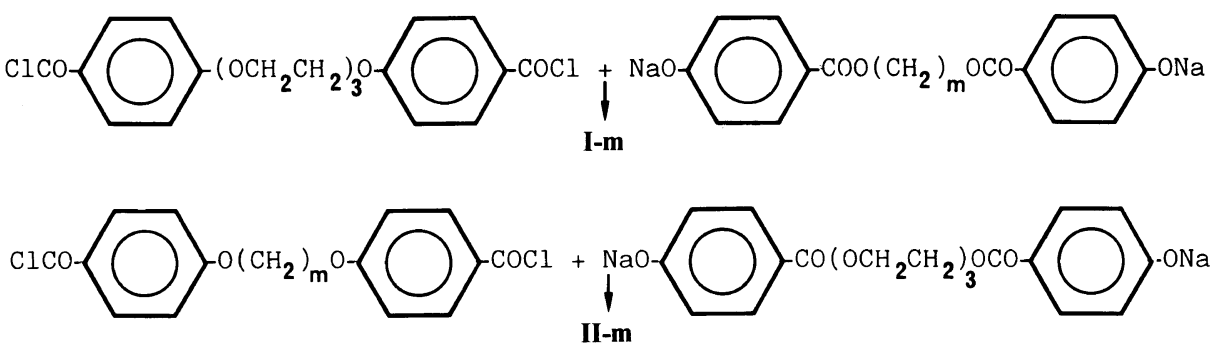

Scheme 2. Synthetic route to the two isomeric twin polyester series I-m and II-m.

Table I. Physicochemical and thermal properties of polyesters I-m and II-m

\begin{tabular}{|c|c|c|c|c|c|c|c|c|}
\hline \multirow{2}{*}{ Sample } & \multirow{2}{*}{$m$} & $|\eta|^{\mathrm{a}}$ & \multirow{2}{*}{$\bar{M}_{n}^{\mathrm{b}}$} & \multirow{2}{*}{$\bar{M}_{w} / \bar{M}_{n}^{\mathrm{b}}$} & \multirow{2}{*}{$\frac{T_{\mathrm{m}}}{{ }^{\circ} \mathrm{C}}$} & \multirow{2}{*}{$\frac{T_{i}}{{ }^{\circ} \mathrm{C}}$} & \multirow{2}{*}{$\frac{\Delta H_{i}}{\mathrm{KJ} \mathrm{mol}^{-1}}$} & \multirow{2}{*}{$\frac{\Delta S_{i}}{\mathrm{~J} \mathrm{~mol}^{-1} \mathrm{~K}^{-1}}$} \\
\hline & & $\mathrm{dl} \mathrm{g}^{-1}$ & & & & & & \\
\hline I-6 & 6 & 0.33 & 8990 & 1.91 & 120 & 177 & 6.66 & 14.8 \\
\hline I-7 & 7 & 0.35 & 7070 & 1.87 & 93 & $-^{c}$ & $-^{c}$ & $-^{c}$ \\
\hline I-8 & 8 & 0.39 & 14090 & 1.93 & 103 & 151 & 8.14 & 19.2 \\
\hline I-9 & 9 & 0.27 & 8810 & 1.95 & 92 & $-^{c}$ & $-^{c}$ & $-^{c}$ \\
\hline I-10 & 10 & 0.32 & 7660 & 2.02 & 114 & 135 & 8.65 & 21.2 \\
\hline I-12 & 12 & 0.34 & 9950 & 1.85 & 120 & 125 & 10.0 & 25.1 \\
\hline II-5 & 5 & 0.56 & 19610 & 2.12 & 135 & $(118)^{d}$ & 1.33 & 3.4 \\
\hline II-6 & 6 & 0.83 & 24320 & 2.05 & 174 & 184 & 4.52 & 9.9 \\
\hline II-7 & 7 & 0.72 & 25130 & 2.22 & 127 & 134 & 1.55 & 3.8 \\
\hline II-8 & 8 & 0.76 & 24480 & 1.93 & 145 & 162 & 6.66 & 15.3 \\
\hline II-9 & 9 & 0.53 & 14140 & 2.02 & 124 & 138 & 1.97 & 4.8 \\
\hline II-10 & 10 & 0.80 & 27820 & 1.73 & 142 & 155 & 8.13 & 19.0 \\
\hline
\end{tabular}

a In chloroform, at $30^{\circ} \mathrm{C}$.

b By GPC, in chloroform.

c Not mesomorphic.

d Monotropic transition.

The mesomorphic properties of the polymers were studied by differential scanning calorimetry, thermal optical analysis and Xray diffraction. The phase transition temperatures together with the relevant enthalpies were obtained from DSC traces on samples annealed by cooling at $10 \mathrm{~K} \mathrm{~min}^{-1}$ from the isotropic melt (Table I).

Polymers of series I-m containing an even number of methylene units show one enantiotropic mesophase, while those having odd values of $m$ do not display mesomorphic properties even in the highly supercooled state. Nematic schlieren textures are observed in the temperature range between melting and iso- tropization, although the high viscosity of the anisotropic melt often hinders the development of well defined textures. The X-ray diffraction patterns obtained for I-6 and I-8 samples at the mesophase temperature only consist of a diffuse outer halo and confirm the nematic nature of the mesophases. On the contrary, all polymers of series H-m exhibit mesomorphic behavior. One mesophase is detected, which is monotropic for sample II-5 and enantiotropic for the other polymers. Optical textures suggest a nematic structure for such mesophases.

The trends of the phase transition temperatures as function of the length of the poly- 
methylene spacer for the two polymer series are reported in Figures 1 and 2. The isotropization temperatures of samples II-m show a distinct even-odd oscillation, even members possessing higher temperatures. While the isotropization temperatures for even members decrease with increasing length of the polymethylene spacer, the odd members follow a slightly rising trend. As a result of these opposite tendencies, the even-odd alternation is extremely pronounced for the lower members but is attenuated as the series is ascended. This unusual behavior has been recently described in several series of poly $(\beta$-aminoester $) \mathrm{s}^{7}$ and poly(ester- $\beta$-sulphide)s. ${ }^{8}$

The isotropization temperatures for even members of series I-m describe a smooth descending profile as the spacer is lengthened. The thermal stability of those members is in any case lower than that of the corresponding even members of series II-m, the difference between the two series increasing with the spacer length from $\Delta T_{i}=7 \mathrm{~K}(m=6)$ to $\Delta T_{i}=20 \mathrm{~K}(m=10)$ (Table I).

Melting temperatures in both polymer series are also characterized by even-odd alternation. While no information is presently available about the crystal structure and the degree of crystallinity of the polymers, it is interesting to note that the melting temperatures of samples II-m are considerably higher than those of the corresponding homologs of series I-m. Accordingly the liquid crystal range, whenever present, results wider for polymers I-m.

In both polymer series the parity of the polymethylene spacer plays an integral role in determining the onset and stability of the liquid crystalline state. The trends of the isotropization entropies for polymers II-m with increasing spacer length (Figure 3) show a marked even-odd oscillation. The isotropization entropies of members with even and odd numbers of methylene units follow two separate and divergent curves, even members giving the upper curve. The isotropization entropies for the even members of series I-m,

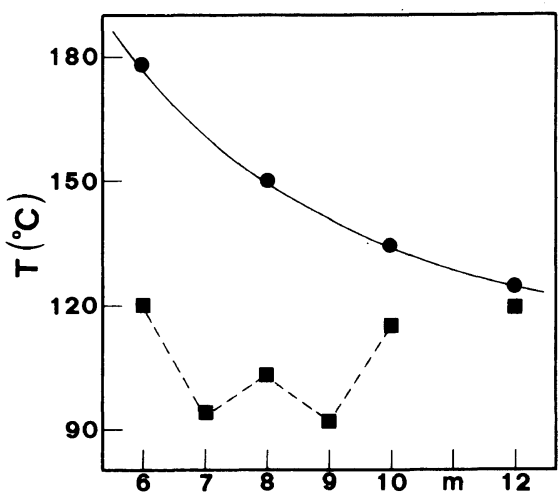

Figure 1. Melting ( $\boldsymbol{\square}$ ) and isotropization (O) temperatures for polyesters I-m as function of the number $m$ of methylene units.

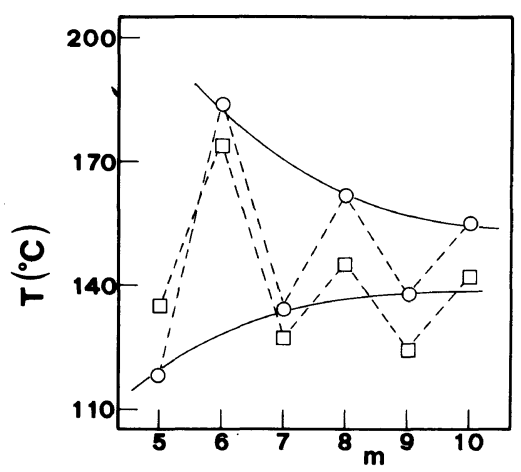

Figure 2. Melting $(\square)$ and isotropization $(O)$ temperatures for polyesters $\mathbf{I I}-\mathrm{m}$ as function of the number $m$ of methylene units.

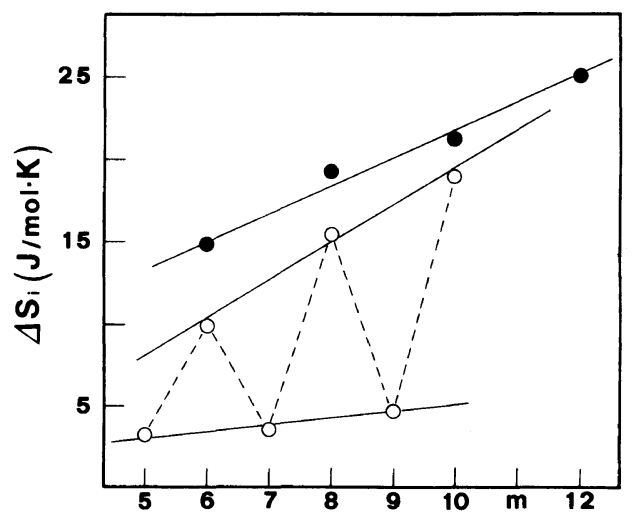

Figure 3. Isotropization entropy for polyesters I-m (O) and II-m (O) as a function of the number $m$ of methylene units. 
plotted vs. spacer length follow a linear trend with a positive slope. By comparing the $\Delta S_{i}$ values for the even members of the two series in the range of polymethylene spacers used, it appears that the mesogen-triethyleneoxidemesogen fragment of even members of series I-m possesses a greater propensity to stabilize the corresponding liquid crystalline state, while the lengthening of the polymethylene segment in the even members of series II-m provides a distinct conformational contribution. Even-odd oscillations of the thermodynamic phase transition parameters have been observed in several series of main chain polymers $^{9-11}$ as well as in low molar mass compounds $s^{12,13}$ and reflect the distinct rotational preferences of polymethylene spacers of different parity. Even members possess ${ }^{14,15}$ a set of extended conformers which can propagate intramolecularly the orientational correlation along the polymer chain enhancing the overall steric and anisotropic interactions and stabilizing the ordered state over the disordered one. On the contrary, the alkylene chain of odd members does not possess the conformational capability of generating prevalent local chain ordering suitable to meet the steric-packing requirements imposed by the liquid crystalline state. The thermodynamic data of the present series I-m and II-m further stress the tendency of even members to strongly participate in determining the order in mesomorphic phases. In recent papers ${ }^{15,16}$ the effect of the orientational placement of the ester function, connecting the mesogenic core with a polymethylene flexible spacer, on the mesophase properties has been investigated using a conformational calculation approach. When the ester function, linking the mesogenic group to the flexible spacer, is assembled as follows $-\mathrm{O}(\mathrm{C}=\mathrm{O})$-spacer-, low energies and high probabilities of existence are associated to the extended conformations of the polymethylene spacer which are prevalently constituted by trans sequences. On the contrary, when the orientational placement of the linking group is inverted $-(\mathrm{O}=\mathrm{C}) \mathrm{O}$-spacer-, the first order interactions relative to the rotation around the $\mathrm{O}-\mathrm{CH}_{2}{ }^{5}-\mathrm{CH}_{2}$ bond favours the gauche over the trans state. Consequently high energies and low probabilities of existence are associated to the extended conformers. In the present series of polymers the unfavorable orientation of the ester linking groups affects separately both the polymethylene and the polyoxyethylene segments. Comparing the thermodynamic behavior in the two series of polymers it appears that the perturbing effect is prevalently operating on the polymethylene spacer (series I-m). In going from series II-m to series I-m, the relative destabilization of the extended conformers of the polymethylene spacers is not fully compensated by the concomitant stabilization of the extended conformers of the polyether segment.

\section{CONCLUSIONS}

The data reported provide a clear proof of the effective role played by the flexible spacers in determining the ultimate properties of semiflexible liquid crystalline polymers relevant to the onset and stability of mesophases. Specific effects can arise not only from the parity of the spacers, but also from the relative position held by the spacer itself within the polymer structural units.

The anisotropy of molecular interactions can either be depressed or even cancelled by subtle changes in the relative orientation of two different flexible spacers with respect to the same mesogenic cores in twin structural units.

Acknowledgement. This work has been carried out with financial support from Ministero Pubblica Istruzione of Italy.

\section{REFERENCES}

1. C. K. Ober, J.-I. Jin, and R. W. Lenz, Adv. Polym. Sci., 59, 103 (1984).

2. A. S. Angeloni, M. Laus, C. Castellari, G. Galli, P. 


\section{A. S. ANGELONi et al.}

Ferruti, and E. Chiellini, Makromol. Chem., 186, 977 (1985).

3. G. Galli, M. Laus, A. S. Angeloni, P. Ferruti, and E. Chiellini, Eur. Polym. J., 21, 727 (1985).

4. A. S. Angeloni, M. Laus, E. Burgin, G. Galli, and E. Chiellini, Polym. Bull., 13, 131 (1985).

5. E. Chiellini, G. Galli, A. S. Angeloni, D. Caretti, and M. Laus, in preparation.

6. G. Galli, E. Chiellini, A. S. Angeloni, and M. Laus, Macromolecules, in the press (1989).

7. M. Laus, A. S. Angeloni, G. Galli, and E. Chiellini, Makromol. Chem., 189, 743 (1988).

8. E. Chiellini, G. Galli, A. S. Angeloni, M. Laus, and R. Pellegrini, Liquid Crystals, 2,529 (1987).

9. A. C. Griffin and S. J. Havens, J. Polym. Sci., Polym.
Phys. Ed., 19, 951 (1981).

10. A. Roviello and A. Sirigu, Makromol. Chem., 183, 895 (1982).

11. A. Blumstein and O. Thomas, Macromolecules, 15, 1264 (1982)

12. J. W. Emsley, G. R. Luckhurst, and G. P. Stockley, Proc. R. Soc. London, Ser. A, 381, 117 (1982).

13. J. A. Buglione, A. Roviello, and A. Sirigu, Mol. Cryst. Liq. Cryst., 106, 169 (1984).

14. A. Abe, Macromolecules, 17, 2280 (1984).

15. D. Y. Yoon and S. Bruckner, Macromolecules, 18, 651 (1985).

16. D. Y. Yoon, S. Bruckner, W. Volksen, J. C. Scott, and A. C. Griffin, Faraday Discuss. Chem. Soc., 79, 41 (1985). 